\title{
Primary Merkel cell carcinoma of the earlobe in a young healthy man
}

\author{
Non Hyeon $\mathrm{Ha}^{1}$, \\ Sue Kyung Kim², \\ Yoo Seob Shin ${ }^{3}$, \\ Sue Min Kim ${ }^{1}$ \\ ${ }^{1}$ Department of Plastic and \\ Reconstructive Surgery, Ajou University \\ Hospital, Suwon; \\ ${ }^{2}$ Department of Dermatology, Seoul \\ Medical Center, Seoul; \\ ${ }^{3}$ Department of Otolaryngology, Ajou \\ University Hospital, Suwon, Korea
}

\begin{abstract}
Merkel cell carcinoma (MCC) is an uncommon neuroendocrine cutaneous tumor with poor prognosis. It has the high rate of recurrence, mortality, regional nodal involvement, and distant metastases. It is difficult to diagnose MCC because of its non-specific clinical findings. It usually occurs on sun-exposed areas of the skin, mostly at head and neck. There is a difference in the incidence and prognosis according to site in the head and neck. However, there is no consented site-specific diagnosis, treatment or follow-up protocol for MCC at the head and neck. We herein report a case of MCC arising in the right earlobe of an otherwise healthy young man who has been diagnosed early, thereby successfully treated. With our closed follow-up, there was no tumor recurrence or complication at 33 months after diagnosis.
\end{abstract}

Keywords: Merkel cell carcinoma / Metastasis / Neuroendocrine tumors

\section{INTRODUCTION}

Merkel cell carcinoma (MCC) is a rare and highly aggressive neuroendocrine cutaneous malignancy [1]. It occurs mostly on sun-exposed areas of the skin in elderly and immunosuppressed individuals $[1,2]$. The average age of diagnosis is 69 years (range, 7 to 104 years) [3-6]. Less than 5\% of cases occur in patients aged $<50$ years; these cases are mostly associated with immunosuppression due to organ transplantation or human immunodeficiency virus infection [1,7]. The most common clinical features of MCC follow the AEIOU (asymptomatic, expanding rapidly, immune suppression, older than 50 years of age, ultraviolet-exposed skin) criteria [8]. More than $90 \%$ of affected patients meet three or more of the AEIOU criteria [8]. However, this diagnostic tool is not specific to MCC; it has been applied to many other cutaneous lesions. Here, we report an uncommon case of MCC arising in the right earlobe of an otherwise healthy young man who fulfilled only two of the AEIOU

Correspondence: Sue Min Kim

Department of Plastic and Reconstructive Surgery, Ajou University Hospital,

164 World cup-ro, Yeongtong-gu, Suwon 16499, Korea

E-mail: rainyhill@gmail.com

Received May 2, 2018 / Revised July 12, 2018 / Accepted July 17, 2018 criteria. Despite only two non-specific symptoms, the patient was diagnosed early and successfully treated.

\section{CASE REPORT}

A 35-year-old man presented to the department of plastic and reconstructive surgery of Ajou University Hospital with a slightly erythematous $1-\mathrm{cm}$-sized mass on his right earlobe; the mass had slowly enlarged during the past 3 months (Fig. 1). The initial clinical impression suggested that the mass was an infected epidermal cyst, and the lesion was therefore completely excised. Histopathological examination revealed uniform and atypical small blue cells arranged in trabecular patterns and sheets (Fig. 2A). The tumor cells contained scanty cytoplasm and irregular nuclei, with several nuclei showing central clearing (Fig. 2B). The tumor cells were positive for cytokeratin 20 (Fig. 3). The mass was diagnosed as MCC.

At this point in time, another 1-cm mass was found in the patient's right infra-auricular area. Computed tomography (CT) and positron emission tomography (PET) of the neck revealed that this lesion was reactive lymphoid hyperplasia rather than metastasis (Fig. 4). A fine-needle aspiration biopsy also demon- 
strated a lack of malignant cells.

According to the National Cancer Care Network (NCCN), wide excision of the primary earlobe lesion was performed with a $1.5-\mathrm{cm}$ safety margin with full thickness, in an attempt to minimize the aesthetic impact and preserve the external acoustic pore. And the defect was reconstructed with local flap advancement. Despite the CT and PET findings, excisional biopsy of the infra-auricular mass was also performed. Histological ex-

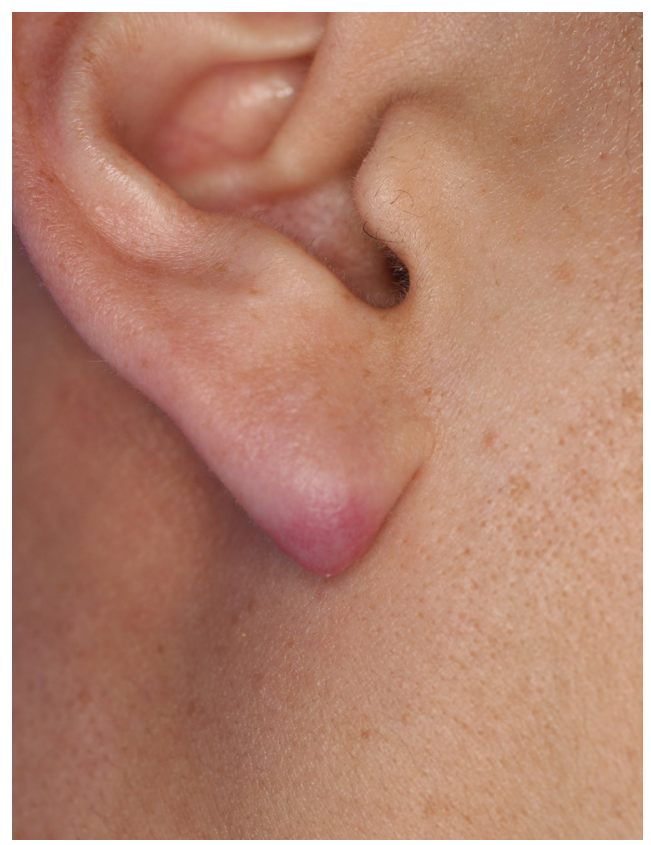

Fig. 1. Gross photograph of the earlobe mass. The mass is $1 \mathrm{~cm}$ in size and displays mild erythema and swelling. amination revealed no residual carcinoma in the excised primary lesion but showed metastatic MCC in the infra-auricular lymph node.

The patient was referred to the ear-nose-throat department and underwent total parotidectomy and supraomohyoid neck dissection. Dissection was done with preserving facial nerve branches. Superficial lobe of parotid gland was partially removed including the mass. Deep lobe of parotid gland was also

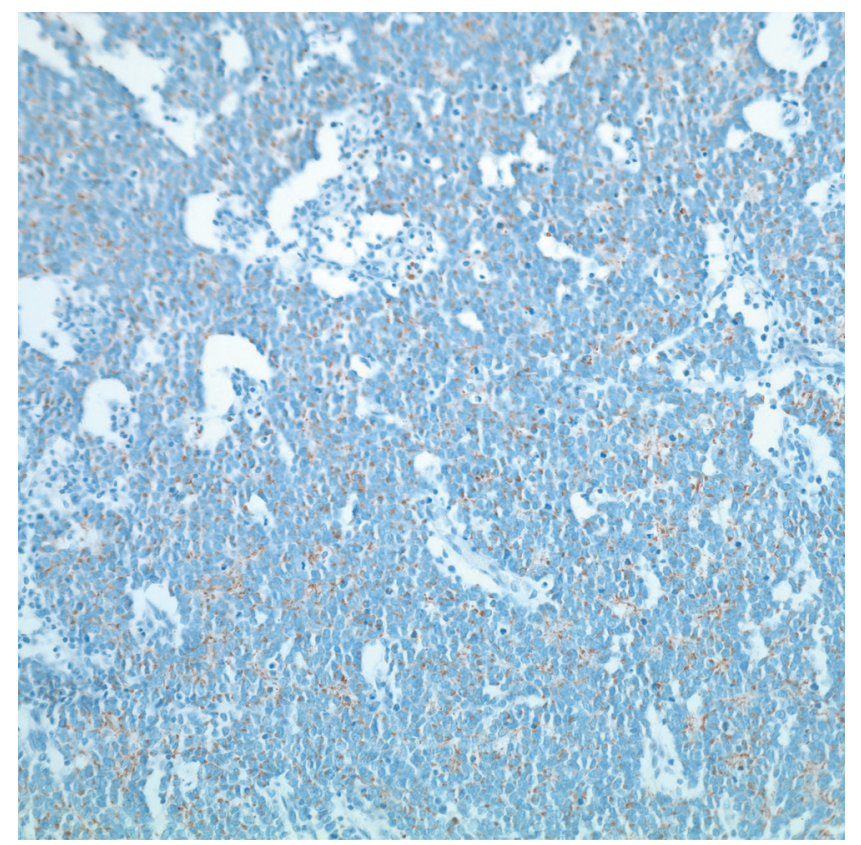

Fig. 3. Immunohistochemistry staining for cytokeratin 20. Tumor cells are positive for cytokeratin $20(\times 200)$.
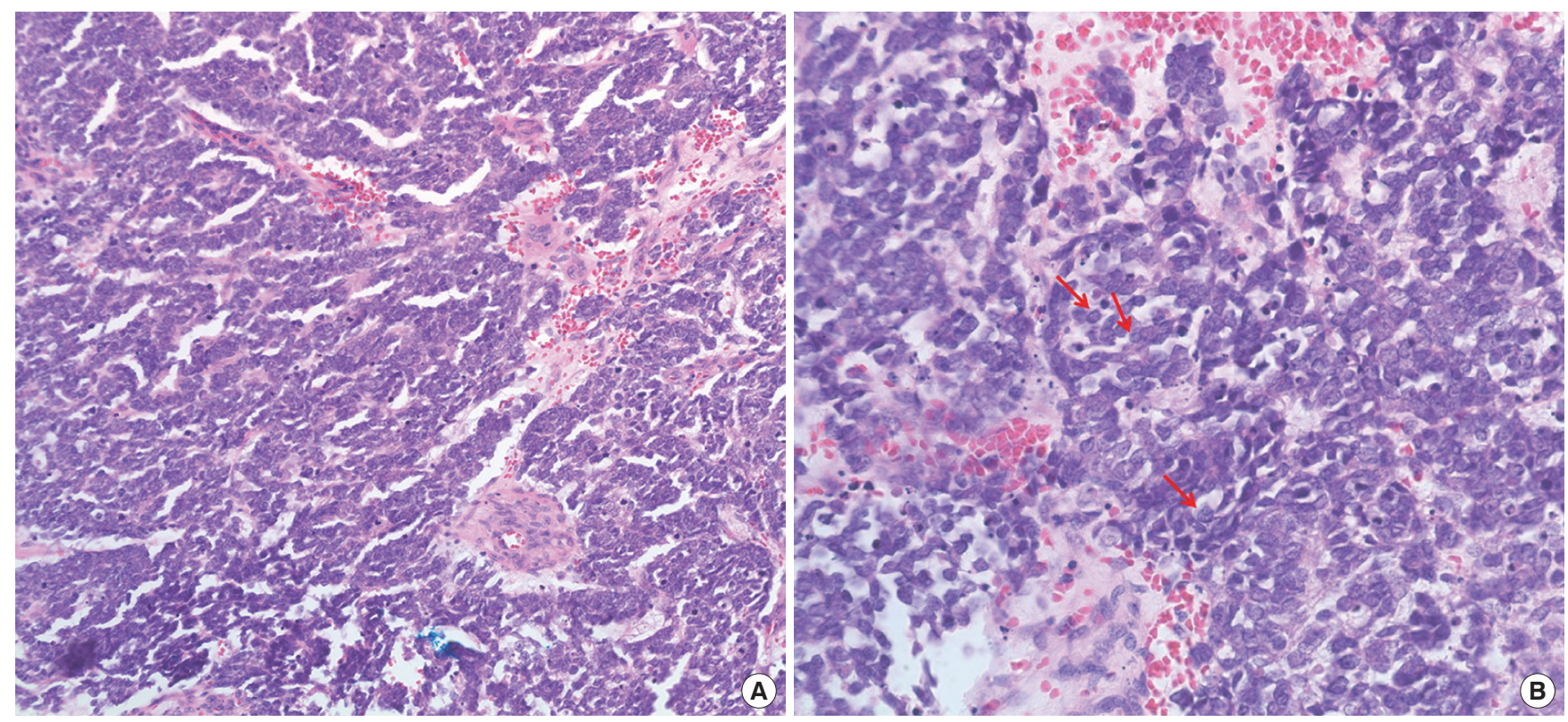

Fig. 2. Histopathological examination of excised mass. (A) Uniform and atypical small blue cells form trabecular pattern and sheets. The tumor cells contain irregular nuclei and scanty cytoplasm $(\mathrm{H} \& \mathrm{E}, \times 200)$. (B) Several nuclei show central clearing (arrows) (H\&E, $\times 400)$. 


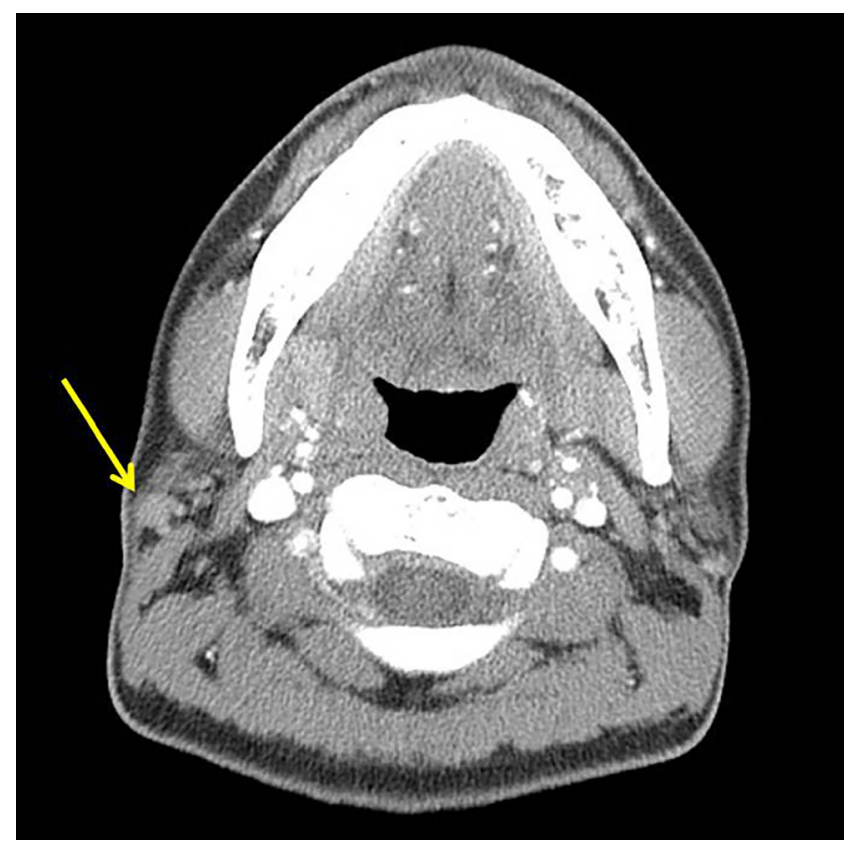

Fig. 4. Computed tomography examination to rule out metastasis. Neck computed tomography shows a 1-cm lymph node at the right intraparotid gland (arrow), suggesting that this is more likely to be reactive hyperplasia than metastasis.

removed. Subplatysmal flap was elevated, and neck level above supraomohyoid lymph nodes were excised. Histological examination revealed no residual carcinoma in the parotid gland or lymph nodes. The patient received no adjuvant radiation or chemotherapy. We followed up the patient every month for first year after diagnosis and followed up every 2 months for second year after diagnosis. After 33 months of follow-up, there was no evidence of tumor recurrence or any other complications (Fig. 5). In present, the patient visits outpatient clinic every 3 or 4 months.

\section{DISCUSSION}

MCC is very rare in young patients; only $5 \%$ of cases occur in patients aged $<50$ years, and in most of these cases, immunosuppression plays a role [2]. Very few cases of MCC arising in young healthy adults have been reported. Three cases of MCC were reported in 15-, 28-, and 33-year-old patients who had undergone chemoradiation or radiotherapy following local wide excision [9-11]. The only other reported cases involve 8and 17-year-old patients who died despite surgery and chemotherapy $[12,13]$. Most of the previous patients with ear primary site were old and died during follow-up [14,15]. Compared with previous cases, it is a very rare case of young male, regional lymph node metastasis in which the patient is alive without recurrence for 33 months after surgery.

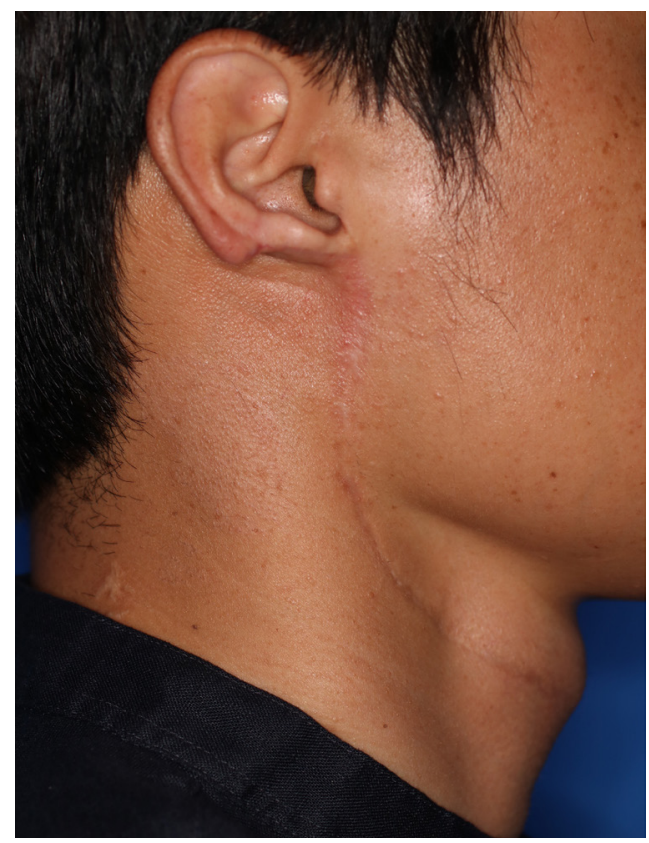

Fig. 5. Thirty-three months postoperative photograph after total parotidectomy and supraomohyoid neck dissection. There was no evidence of tumor recurrence or any other complications.

Because MCC is rare, established treatments are often based on retrospective series; no prospective trials have been performed to investigate the effects of surgery, radiotherapy, or chemotherapy. Wide tumor resection is usually recommended with a 1- to 2-cm tumor-free margin, and complete lymph node dissection is recommended in patients with clinically node-positive MCC [16]. We performed wide excision of the primary lesion with a $1.5-\mathrm{cm}$ safety margin in an attempt to minimize the aesthetic impact and preserve the external acoustic pore. Although lymph node metastasis was pathologically confirmed, all specimens from the lymph node dissection tested negative for cancer cells. MCC has an aggressive course with early lymphatic and hematogenous spread. Therefore, postoperative radiotherapy is generally recommended even in cases of negative margins [17]. In the present case, we achieved local control of both the primary lesion and metastatic disease. The patient was not administered any adjuvant therapy, but was closely observed. The patient was doing well after 33 months of follow-up. Our patient was properly treated because of the early diagnosis; he visited our clinic soon after recognizing the mass, and excisional biopsy was immediately performed. Although no metastatic nodes were found on the CT and PET images or fine-needle aspiration biopsy results, we performed excisional biopsy because of the development of the new palpable lesion and in consideration of the aggressive nature of MCC. We determined that early diagnosis with a high suspicion for metasta- 


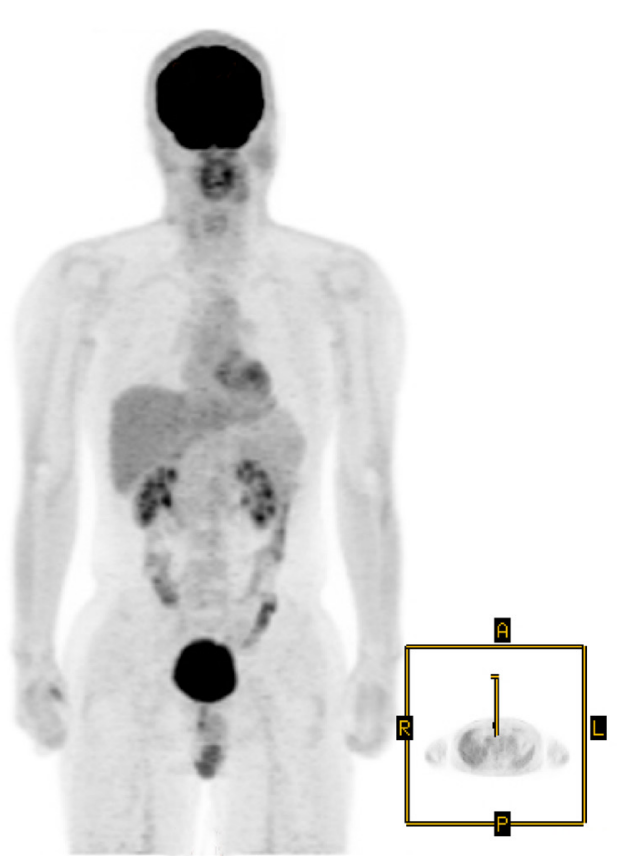

Fig. 6. Positron emission tomography-computed tomography examination for detecting local recurrence or distant metastasis 2 years after surgery. No significant abnormal fludeoxyglucose uptake to suggest malignancy.

sis was critical for successful treatment in this case.

No consented aftercare protocols exist for MCC. MCC recurrence presents within 3 years of diagnosis in $90 \%$ of cases. The NCCN recommendations call for follow-up visits every 3 to 6 months in the first 2 years after diagnosis and later every 6 to 12 months [18]. During the follow-up period after diagnosis, every visit included a physical examination and thorough palpation of lymph nodes. Magnetic resonance imaging was carried out for confirming the local tumor recurrence and cervical lymph node metastasis every year. Also, PET-CT was carried out for detecting local recurrence and distant metastasis 2 years after surgery (Fig. 6). As a result, there was no evidence of local tumor recurrence, cervical lymph node metastasis or distant metastasis in imaging studies.

MCC most commonly occurs in the head and neck region due to chronic sun exposure. Smith et al. [19] reported the relationship between tumor location and prognosis among patients with MCC of the head and neck. Tumors are most commonly located on the face (61.1\%), followed by the scalp/neck (21.2\%), ear (7.0\%), eyelid (5.4\%), and lip (5.3\%). Ear tumors had the highest rate of nodal metastasis (63.2\%) and lip tumor had highest rate of invasion into bone, cartilage, and muscle (13.7\%). Patients with scalp/neck and lip primary sites had significantly worse survival rate relative to patients with other location [19]. Although there was the variability of survival rates depending on data collection, the 5 -year overall survival for pa- tients who had regional lymph node involvement without disseminated metastatic disease was almost 40\% [1].

Because MCC lacks specific presenting clinical features, it is important to cautiously examine the findings using a wide range of clinical tools to achieve an accurate histopathologic diagnosis. Histopathological findings of MCC resemble those of other small round blue cell tumors such as primitive neuroectodermal tumors, metastatic small cell carcinoma, lymphoma/ leukemia, alveolar rhabdomyosarcoma, and melanoma. Differential diagnosis is possible by immunohistochemical staining with the sensitive and specific marker cytokeratin 20. Additionally, most cases of MCC are positive for neurofilaments and the neuroendocrine markers chromogranin A, synaptophysin, and neuron-specific enolase but negative for thyroid transcription factor 1, CD20, CD3, myeloperoxidase, desmin, and S100. Using these markers, other neoplasms can be distinguished from MCC.

In summary, we have herein reported a case of primary MCC in a young healthy man, achieving early diagnosis and successful treatment. Considering that clinical diagnosis is very difficult in MCC, early histopathologic diagnosis with high suspicion is crucial for early diagnosis and proper management.

\section{CONFLICT OF INTEREST}

No potential conflict of interest relevant to this article was reported.

\section{PATIENT CONSENT}

The patients provided written informed consent for the publication and the use of their images.

\section{REFERENCES}

1. Schadendorf D, Lebbe C, Zur Hausen A, Avril MF, Hariharan S, Bharmal M, et al. Merkel cell carcinoma: epidemiology, prognosis, therapy and unmet medical needs. Eur J Cancer 2017;71: 53-69.

2. Pectasides D, Pectasides M, Economopoulos T. Merkel cell cancer of the skin. Ann Oncol 2006;17:1489-95.

3. Ott MJ, Tanabe KK, Gadd MA, Stark P, Smith BL, Finkelstein DM, et al. Multimodality management of Merkel cell carcinoma. Arch Surg 1999;134:388-92.

4. Boyle F, Pendlebury S, Bell D. Further insights into the natural history and management of primary cutaneous neuroendocrine (Merkel cell) carcinoma. Int J Radiat Oncol Biol Phys 1995;31:315-23. 
5. Smith DF, Messina JL, Perrott R, Berman CG, Reintgen DS, Cruse CW, et al. Clinical approach to neuroendocrine carcinoma of the skin (Merkel cell carcinoma). Cancer Control 2000;7: 72-83.

6. Schmid C, Beham A, Feichtinger J, Aubock L, Dietze O. Recurrent and subsequently metastasizing Merkel cell carcinoma in a 7-year-old girl. Histopathology 1992;20:437-9.

7. Buell JF, Trofe J, Hanaway MJ, Beebe TM, Gross TG, Alloway $\mathrm{RR}$, et al. Immunosuppression and Merkel cell cancer. Transplant Proc 2002;34:1780-1.

8. Heath M, Jaimes N, Lemos B, Mostaghimi A, Wang LC, Penas PF, et al. Clinical characteristics of Merkel cell carcinoma at diagnosis in 195 patients: the AEIOU features. J Am Acad Dermatol 2008;58:375-81.

9. Roy S, Das I, Nandi A, Roy R. Primary Merkel cell carcinoma of the oral mucosa in a young adult male: report of a rare case. Indian J Pathol Microbiol 2015;58:214-6.

10. Prabhu S, Smitha RS, Punnya VA. Merkel cell carcinoma of the alveolar mucosa in a young adult: a rare case report. Br J Oral Maxillofac Surg 2010;48:48-50.

11. Halvorson CR, McClain S, Rady P, Tyring S, Gaspari AA. Merkel cell carcinoma presenting in a young patient as a forme fruste of variant epidermodysplasia verruciformis. J Am Acad Dermatol 2011;65:667-9.
12. Koksal Y, Toy H, Talim B, Unal E, Akcoren Z, Cengiz M. Merkel cell carcinoma in a child. J Pediatr Hematol Oncol 2009;31:359-61.

13. Marzban S, Geramizadeh B, Farzaneh MR. Merkel cell carcinoma in a 17-year-old boy, report of a highly aggressive fatal case and review of the literature. Rare Tumors 2011;3:e34.

14. Rinaldo A, Devaney KO, Ferlito A. Merkel cell carcinoma of the auricle. Acta Otolaryngol 2005;125:125-9.

15. Day KE, Carroll WR, Rosenthal EL. Parotid gland metastasis in Merkel cell carcinoma of the head and neck: a series of 14 cases. Ear Nose Throat J 2016;95:398-404.

16. Schwartz JL, Wong SL, McLean SA, Hayman JA, Lao CD, Kozlow JH, et al. NCCN guidelines implementation in the multidisciplinary Merkel Cell Carcinoma Program at the University of Michigan. J Natl Compr Canc Netw 2014;12:434-41.

17. Eich HT, Eich D, Staar S, Mauch C, Stutzer H, Groth W, et al. Role of postoperative radiotherapy in the management of Merkel cell carcinoma. Am J Clin Oncol 2002;25:50-6.

18. Llombart B, Kindem S, Chust M. Merkel cell carcinoma: an update of key imaging techniques, prognostic factors, treatment, and follow-up. Actas Dermosifiliogr 2017;108:98-107.

19. Smith VA, MaDan OP, Lentsch EJ. Tumor location is an independent prognostic factor in head and neck Merkel cell carcinoma. Otolaryngol Head Neck Surg 2012;146:403-8. 